\title{
Asymmetric lasing at spectral singularities
}

\author{
L. Jin* \\ School of Physics, Nankai University, Tianjin 300071, China
}

(Received 18 January 2018; published 20 March 2018)

\begin{abstract}
Scattering coefficients can diverge at spectral singularities. In such situation, the stationary solution becomes a laser solution with outgoing waves only. We explore a parity-time $(\mathcal{P} \mathcal{T})$-symmetric non-Hermitian two-arm Aharonov-Bohm interferometer consisting of three coupled resonators enclosing synthetic magnetic flux. The synthetic magnetic flux does not break the $\mathcal{P} \mathcal{T}$ symmetry, which protects the symmetric transmission. The features and conditions of symmetric, asymmetric, and unidirectional lasing at spectral singularities are discussed. We elucidate that lasing affected by the interference is asymmetric; asymmetric lasing is induced by the interplay between the synthetic magnetic flux and the system's non-Hermiticity. The product of the left and right transmissions is equal to that of the reflections. Our findings reveal that the synthetic magnetic flux affects light propagation, and the results can be applied in the design of lasing devices.
\end{abstract}

DOI: 10.1103/PhysRevA.97.033840

\section{INTRODUCTION}

The theory of parity-time $(\mathcal{P} \mathcal{T})$-symmetric systems has been extensively investigated [1-15]. In experiments, the use of optical platforms is fruitful for investigating $\mathcal{P} \mathcal{T}$ symmetric non-Hermitian quantum mechanics. To construct $\mathcal{P} \mathcal{T}$-symmetric systems, passive and active devices are mostly used. In passive systems, elements have varying loss rates [16]. A passive system is equivalently described by a $\mathcal{P} \mathcal{T}$-symmetric Hamiltonian after an overall loss rate has been removed; however, mode amplification is impossible in passive systems. In active systems, the gain balances the loss to form a $\mathcal{P} \mathcal{T}$ symmetric system [17-21]. Under steady-state conditions, a resonator doped with erbium ions under pumping induces a gain that balances the loss in the dissipative resonator. Active $\mathcal{P} \mathcal{T}$-symmetric devices have numerous applications such as in optical isolators [20] and single-mode $\mathcal{P} \mathcal{T}$-symmetric lasing $[22,23]$.

The non-Hermiticity induces asymmetric and nonunitary scattering in non-Hermitian systems [24,25]. Numerous intriguing phenomena such as unidirectional reflectionless $[26,27]$, invisible cloaking $[28,29]$ and asymmetric and robust light transport have been reported [30,31]. Spectral singularities exist in the non-Hermitian scattering system, where the scattering coefficient diverges and the completeness of eigenstates is spoiled [32-36]. At spectral singularities, coherent perfect absorption [37-39] and a $\mathcal{P} \mathcal{T}$-symmetric laser absorber [40-42] have been demonstrated.

In Hermitian systems, symmetric scattering exists even in the presence of synthetic magnetic flux, where photons

\footnotetext{
*jinliang@nankai.edu.cn

Published by the American Physical Society under the terms of the Creative Commons Attribution 4.0 International license. Further distribution of this work must maintain attribution to the author(s) and the published article's title, journal citation, and DOI.
}

mimic electrons in magnetic field [43-49]. The synthetic magnetic flux induces asymmetric couplings but does not break the time-reversal symmetry. The time-reversal symmetry protects the optical reciprocity and the symmetric scattering. However, in non-Hermitian systems, the synthetic magnetic flux and the non-Hermiticity can induce novel asymmetric behaviors [50]. The synthetic magnetic flux enclosed in a loss resonator side-coupled to a resonator chain helps to create a unidirectional perfect absorber in which light can avoid occupation of the loss resonator. The proposed system has full reflectionless absorption in one direction and full reflectionless transmission in the opposite direction [51-54]. In contrast to a perfect absorber, a $\mathcal{P} \mathcal{T}$-symmetric laser at non-Hermitian spectral singularities, where symmetrical lasing toward both sides of a scattering system occurs, has been demonstrated $[40,41,55]$. Moreover, unidirectional lasing at unidirectional spectral singularities has been proposed [56].

In this paper, we focus on a $\mathcal{P} \mathcal{T}$-symmetric non-Hermitian system consisting of three coupled optical resonators and in which synthetic magnetic flux is enclosed. The three coupled resonators are embedded in a resonator array and collectively serve as an Aharonov-Bohm (AB) interferometer. The nonHermiticity of the system is due to the balanced gain and loss in the resonators. Synthetic magnetic flux is induced through an asymmetric coupling between neighboring resonators. $\mathcal{P} \mathcal{T}$ symmetry holds in the presence of synthetic magnetic flux and enables symmetric light transmission even though the coupling is asymmetric. The scattering coefficients diverge at spectral singularities of the non-Hermitian interferometer, with the scattering wave function in the steady state representing lasing. The features and conditions of symmetric, asymmetric, and unidirectional lasing at spectral singularities are discussed in this paper. The synthetic magnetic flux affects the light interference and leads to asymmetric lasing in the interferometer. As an illustration, we evaluate the scattering properties of a uniformly coupled resonator system. At spectral singularities, lasing asymmetry is minimal at trivial synthetic 


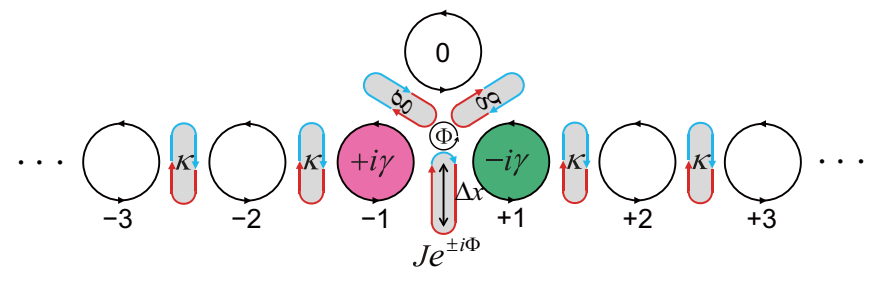

FIG. 1. Schematic of the coupled resonators AB interferometer. Uniformly coupled resonator array with the embedded three coupled resonator. The gain (pink) and loss (green) in the resonators are balanced. The passive resonators are in white. The primary resonators (ring shape) are coupled through the auxiliary resonators (stadium shape). The photons circling in the primary and auxiliary resonators are in opposite directions.

magnetic flux and maximal at half-integer synthetic magnetic flux. Wave emission has the signature of spectral singularities in the wave-packet scattering process.

The remainder of this paper is organized as follows. In Sec. II, we model the $\mathcal{P} \mathcal{T}$-symmetric coupled resonator $A B$ interferometer. In Sec. III, we describe its spectral singularities and asymmetric lasing. In Sec. IV, we consider a uniformly coupled resonator as an illustration. Finally, we summarize our study in Sec. V.

\section{II. $\mathcal{P} \mathcal{T}$-SYMMETRIC AB INTERFEROMETER}

Photons do not directly interact with magnetic fields; however, the photonic analogy of the AB effect is realized through various methods such as dynamic modulation of material permittivity, photon-phonon interaction, and magneto-optical effects [43-46]. An AB interferometer is depicted in Fig. 1. The ring-shaped resonators are the primary resonators; they are coupled through the stadium-shaped auxiliary resonators [57]. The AB interferometer consists of two uniformly coupled passive ring resonator arrays and three ring resonators in the center. The primary resonator frequency is $\omega_{\mathrm{c}}$. Resonator +1 has loss (green in Fig. 1), resonator -1 has gain (pink in Fig. 1), and resonator 0 is passive (white in Fig. 1). The resonators are evanescently coupled in a ring configuration and embedded in the resonator array between resonators +2 and -2 . The coupling of the uniform resonator array is $-\kappa$. The loss is caused by resonator dissipation, whereas the gain is induced by pumping the ions doped in resonator -1 . The gain is modeled by a constant rate $\gamma$ when the gain is not close to saturation in the linear region $[18,22]$. The couplings between resonators 0 and \pm 1 are $-g$. The resonators $-1,0$, and +1 compose the scattering center $H_{\mathrm{c}}$.

The system is a two-arm $\mathrm{AB}$ interferometer. One arm comprises the indirect path from resonator -1 to resonator +1 through resonator 0 ; the coupling strengths are $-g$. The other arm of the interferometer comprises the direct path from resonator -1 to resonator +1 ; the coupling between resonators -1 and +1 has a directional hopping phase, represented by $-J e^{ \pm i \Phi}$ [57]. This asymmetric coupling is introduced using the optical path imbalance method illustrated in Fig. 1. Notably, $\Phi=2 \pi \Delta x / \lambda$ depends on the path-length difference $2 \Delta x$ experienced by photons in the auxiliary resonator as they travel between resonators -1 and +1 in opposite directions (Fig. 1), where $\lambda$ is the optical wave length. The resonator supports clockwise and counterclockwise modes; the black arrows in Fig. 1 represent the counterclockwise mode. The cyan (red) arrows indicate the optical path lengths of photons tunneling from left to right (right to left). Photons tunneling between resonators -1 and +1 feel a path difference $2 \Delta x$, as illustrated in Fig. 1; this path difference results in an additional phase factor $e^{ \pm i \Phi}$ in the coupling process. The additional directional phase factor corresponds to synthetic magnetic flux $\Phi$ that is enclosed by the three coupled resonators [58]. The synthetic magnetic flux is gauge invariant. Although the transmission and reflection coefficients change, their moduli are invariant under gauge transformation.

In the clockwise mode, photons circle in the opposite direction and all arrows in Fig. 1 are invert. The path differences in the tunneling process are opposite for the counterclockwise and clockwise modes. Consequently, opposite synthetic magnetic fluxes $\Phi$ and $-\Phi$ are induced. The synthetic magnetic flux $\Phi$ and its opposite $-\Phi$ lead to identical transmission and reflection probabilities. Therefore, the lasing at spectral singularities is identical for the counterclockwise and clockwise modes.

The modal amplitudes of the resonators are described by coupled-mode theory [59], whereas the equation of motion for the leads is

$$
i \dot{a}_{j}=\omega_{\mathrm{c}} a_{j}-\kappa a_{j-1}-\kappa a_{j+1},
$$

for the left lead $j<-1$ and the right lead $j>1$. The equations of motion for the scattering center are

$$
\begin{aligned}
i \dot{a}_{-1} & =\left(\omega_{\mathrm{c}}+i \gamma\right) a_{-1}-g a_{0}-J e^{i \Phi} a_{1}-\kappa a_{-2}, \\
i \dot{a}_{0} & =\omega_{\mathrm{c}} a_{0}-g a_{-1}-g a_{1}, \\
i \dot{a}_{1} & =\left(\omega_{\mathrm{c}}-i \gamma\right) a_{1}-g a_{0}-J e^{-i \Phi} a_{-1}-\kappa a_{2} .
\end{aligned}
$$

The equations of motion are Schrödinger-like equations. The dynamics of photons in the synthetic magnetic flux described by these equations of motion are equivalent to those of electrons in magnetic flux in condensed-matter physics.

Notably, the interferometer is $\mathcal{P} \mathcal{T}$ symmetric in the presence of synthetic magnetic flux. The parity operator $\mathcal{P}$ satisfies $\mathcal{P} \hat{a}_{-j}^{\dagger} \mathcal{P}^{-1}=\hat{a}_{j}^{\dagger}$ and $\mathcal{P} \hat{a}_{j} \mathcal{P}^{-1}=\hat{a}_{-j}$. The time-reversal operator $\mathcal{T}$ satisfies $\mathcal{T} i \mathcal{T}^{-1}=-i . \hat{a}_{j}^{\dagger}\left(\hat{a}_{j}\right)$ is the creation (annihilation) operator for the resonator $j$.

\section{SPECTRAL SINGULARITIES AND LASING}

In a finite-size $\mathcal{P} \mathcal{T}$-symmetric non-Hermitian system, the system might experience a phase transition from an exact $\mathcal{P} \mathcal{T}$-symmetric phase to a broken- $\mathcal{P} \mathcal{T}$-symmetric phase at the exceptional points. The exceptional points are points that the finite-size non-Hermitian system is defective, where coalesced eigenstates appear. Another type of singularity is spectral singularities in infinite-size non-Hermitian systems. Spectral singularities are singular points in a non-Hermitian scattering system at which eigenstates for a continuous spectrum are incomplete [32]. The biorthonormal basis vanishes at the singularities of non-Hermitian systems [60]. In non-Hermitian optical scattering systems, coherent perfect absorption or lasing occurs at the spectral singularities of $\mathcal{P} \mathcal{T}$-symmetric systems [37-41]. The stationary scattering wave function is that of an incoming wave for a perfect absorber, where outgoing 
(a)

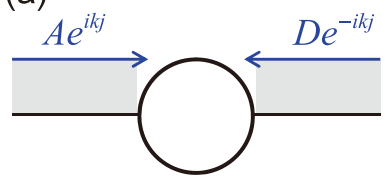

(c)

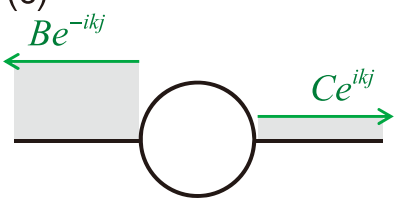

(b)

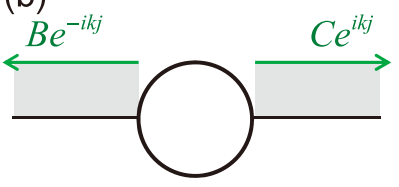

(d)

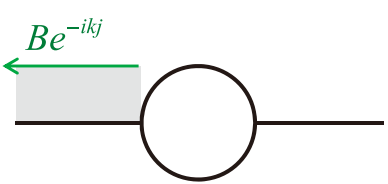

FIG. 2. The coherent perfect absorbing and lasing. The circles indicate the non-Hermitian structures. The incoming (outgoing) waves are indicated by the blue (green) arrows. (a) Coherent perfect absorbing, (b) symmetric lasing, (c) asymmetric lasing, (d) unidirectional lasing.

waves vanish [Fig. 2(a)]. In contrast, lasing corresponds to a stationary scattering wave function that constitutes outgoing waves only [Figs. 2(b)-2(d)]. Unidirectional perfect absorption has been proposed at spectral singularities in dissipative systems that are non- $\mathcal{P} \mathcal{T}$ symmetric [52-54]. The $\mathcal{P} \mathcal{T}$-symmetric scattering system considered in this paper is a non-Hermitian two-arm $\mathrm{AB}$ interferometer. We are interested in how spectral singularities and lasing are affected by the synthetic magnetic flux that causes interference between the two arms.

The dispersion relation for the coupled resonators is $\varepsilon=$ $\omega_{\mathrm{c}}-2 \kappa \cos k$, where $k$ is the wave vector, $-\pi \leqslant k \leqslant \pi$. The modal amplitude satisfies $a_{j}=f_{j} e^{-i \varepsilon t}$. From the equations of motion (2)-(4), we have

$$
\begin{aligned}
& -g f_{0}-J e^{i \Phi} f_{1}-\kappa f_{-2}=(E-i \gamma) f_{-1}, \\
& -g f_{-1}-g f_{1}=E f_{0}, \\
& -g f_{0}-J e^{-i \Phi} f_{-1}-\kappa f_{2}=(E+i \gamma) f_{1},
\end{aligned}
$$

and $-f_{j-1}-f_{j+1}=E f_{j}$ for $|j|>1$, where $E=-2 \kappa \cos k$. In the elastic scattering process, the wave functions $f_{j}$ at the steady state are in the form of $f_{j}=A e^{i k j}+B e^{-i k j}(j<-1)$ and $f_{j}=C e^{i k j}+D e^{-i k j}(j>1)$, where $A$ and $D$ are the amplitudes of the incoming wave, and $B$ and $C$ are the amplitudes of the outgoing wave.

At spectral singularities, the product of the left and right transmissions is equal to that of the reflections; in other words, $T_{\mathrm{L}} T_{\mathrm{R}}=R_{\mathrm{L}} R_{\mathrm{R}}$ holds, where $T(R)$ represents the transmission (reflection) probability. The subscripts indicate either the left or right input. The scattering coefficients diverge at spectral singularities for lasing and the wave function consists of outgoing waves only,

$$
f_{j}= \begin{cases}B e^{-i k j} & (j<0) \\ C e^{+i k j} & (j>0),\end{cases}
$$

where incoming waves $A e^{i k j}(j<0)$ and $D e^{-i k j}(j>0)$ vanish. For a $\mathcal{P} \mathcal{T}$-symmetric interferometer, the transmission or reflection probability is symmetric. Therefore, symmetric lasing of a $\mathcal{P} \mathcal{T}$-symmetric interferometer requires not only symmetric reflection and transmission probabilities, but also that these probabilities possess identical values or, in other words, $T_{\mathrm{L}}=T_{\mathrm{R}}=R_{\mathrm{L}}=R_{\mathrm{R}}$ [Fig. 2(b)]; otherwise, the lasing

is asymmetric [Fig. 2(c)]. $T_{\mathrm{L}} T_{\mathrm{R}}=R_{\mathrm{L}} R_{\mathrm{R}}$ implies asymmetric lasing with contrast $\chi=T_{\mathrm{L}} / R_{\mathrm{L}}=R_{\mathrm{R}} / T_{\mathrm{R}}$.

The enclosed synthetic magnetic flux affects the interference and spectral singularities. By substituting $f_{-2}=B e^{i k}$, $f_{-1}=B, f_{1}=C$, and $f_{2}=C e^{i k}$ into the equations of motion [Eqs. (5)-(7)] and then eliminating $f_{0}$, we obtain

$$
\begin{aligned}
& \left(\frac{g^{2}}{E}-J e^{i \Phi}\right) C=\left(E+\kappa e^{i k}-\frac{g^{2}}{E}-i \gamma\right) B, \\
& \left(\frac{g^{2}}{E}-J e^{-i \Phi}\right) B=\left(E+\kappa e^{i k}-\frac{g^{2}}{E}+i \gamma\right) C,
\end{aligned}
$$

where $E=-2 \kappa \cos k$. When $B$ and $C$ are nonvanishing, by eliminating $B$ and $C$ from Eqs. (9) and (10), we get

$$
\left(\kappa e^{-i k}+\frac{g^{2}}{E}\right)^{2}+\gamma^{2}=\left(\frac{g^{2}}{E}-J e^{i \Phi}\right)\left(\frac{g^{2}}{E}-J e^{-i \Phi}\right) .
$$

After simplifying this relation [Eq. (11)], we obtain the lasing conditions at spectral singularities as follows:

$$
\begin{gathered}
g^{2}=2 \kappa^{2} \cos ^{2} k, \\
\cos \Phi=\frac{\gamma^{2}-J^{2}-\kappa^{2}}{g^{2}} \frac{\kappa}{J} \cos k .
\end{gathered}
$$

At spectral singularities, the wave function consists of outgoing waves. The modal amplitudes satisfy $|B|^{2} /|C|^{2}=$ $(\gamma+\kappa \sin k) /(\gamma-\kappa \sin k)$, which indicates that the lasing is asymmetric in the setup of the $\mathcal{P} \mathcal{T}$-symmetric interferometer.

An extreme case of asymmetric lasing occurs at unidirectional spectral singularities [56]. The lasing occurs in one direction only, where $B$ or $C$ vanishes in the scattering wave function $f_{j}$ [Fig. 2(d)]. In this case, Eqs. (9) and (10) reduce to

$$
\begin{gathered}
\frac{g^{2}}{2 \kappa \cos k}+J \cos \Phi+i \sigma J \sin \Phi=0, \\
\kappa \cos k-\frac{g^{2}}{2 \kappa \cos k}-i(\kappa \sin k+\sigma \gamma)=0,
\end{gathered}
$$

with $\sigma=+1(-1)$ for $B=0(C=0)$. Equations (14) and (15) indicate that the unidirectional spectral singularities place additional constraints on the parameters of the coupled resonator system. The real and imaginary parts of the left side of Eqs. (14) and (15) vanish, respectively.

Equation (14) yields that the synthetic magnetic flux must be

$$
\Phi=n \pi \quad(n \in \mathbb{Z}) .
$$

Equation (15) yields

$$
\gamma^{2}=\kappa^{2} \sin ^{2} k, g^{2} / 2=\kappa^{2} \cos ^{2} k .
$$

By associating Eqs. (14) and (15), we notice that the coupling, the synthetic magnetic flux, and the gain or loss rate are related to the wave vector as follows:

$$
J \cos \Phi=-\kappa \cos k .
$$

Notably, $\cos ^{2} \Phi=1$ for $\Phi=n \pi,(n \in \mathbb{Z})$. Thus, the coupling strengths satisfy $g^{2}=2 J^{2}$ and the parameters take the forms of a circle and an ellipse in parameter space,

$$
J^{2}+\gamma^{2}=\kappa^{2}
$$


and

$$
g^{2} / 2+\gamma^{2}=\kappa^{2}
$$

At unidirectional spectral singularities, the symmetric transmission is finite; the reflection is infinite for one input direction and zero for the other input direction. The stationary wave function for $\gamma=\kappa \sin k$ is

$$
f_{j}= \begin{cases}e^{-i k j} & (j<0) \\ 0 & (j>0)\end{cases}
$$

and the stationary wave function for $\gamma=-\kappa \sin k$ is

$$
f_{j}= \begin{cases}0 & (j<0) \\ e^{+i k j} & (j>0)\end{cases}
$$

The stationary wave function for unidirectional lasing implies that the lasing is directional, and this directionality is generated from the gain resonator as a reflection toward the lead to which it is connected. By contrast, the reflection in the other direction is perfectly absorbed by the loss resonator. The clockwise and counterclockwise modes experience opposite synthetic magnetic fluxes. From the conditions of spectral singularities, we notice that they both lase at spectral singularities for fixed device parameters.

\section{UNIFORMLY COUPLED $\mathcal{P} \mathcal{T}$-SYMMETRIC AB INTERFEROMETER}

As an illustrative example, we investigate the $\mathrm{AB}$ interferometer in the case of $J=g=\kappa$. For the left input with wave vector $k$, the scattering wave function in the left lead is $f_{j}=e^{i k j}+r_{\mathrm{L}} e^{-i k j}(j<-1)$ and the transmission wave in the right lead is $f_{j}=t_{\mathrm{L}} e^{i k j}(j>1)$. By contrast, for the right input, the input wave function in the right lead is $f_{j}=e^{-i k j}+r_{\mathrm{R}} e^{i k j}(j>1)$ and the transmission wave in the left lead is $f_{j}=t_{\mathrm{R}} e^{-i k j}(j<-1) . t_{\mathrm{L}(\mathrm{R})}\left(r_{\mathrm{L}(\mathrm{R})}\right)$ represents the transmission (reflection) coefficient for the left (right) input. The transmission and reflection probabilities are $T_{\mathrm{L}(\mathrm{R})}=$ $\left|t_{\mathrm{L}(\mathrm{R})}\right|^{2}$ and $R_{\mathrm{L}(\mathrm{R})}=\left|r_{\mathrm{L}(\mathrm{R})}\right|^{2}$. Substituting the wave function $f_{j}$ into the equations of motion, the scattering coefficients $r_{\mathrm{L}, \mathrm{R}}$ and $t_{\mathrm{L}, \mathrm{R}}$ are obtained as follows:

$$
\begin{aligned}
t_{\mathrm{L}} & =\frac{i \sin k\left(1+e^{-i \Phi} 2 \cos k\right)}{i e^{-2 i k} \sin k+\left(1-\gamma^{2}\right) \cos k+\cos \Phi}, \\
r_{\mathrm{L}} & =\frac{\gamma \sin (2 k)-\left(1-\gamma^{2}\right) \cos k-\cos \Phi}{i e^{-2 i k} \sin k+\left(1-\gamma^{2}\right) \cos k+\cos \Phi}, \\
t_{\mathrm{R}} & =\frac{i \sin k\left(1+e^{i \Phi} 2 \cos k\right)}{i e^{-2 i k} \sin k+\left(1-\gamma^{2}\right) \cos k+\cos \Phi}, \\
r_{\mathrm{R}} & =\frac{-\gamma \sin (2 k)-\left(1-\gamma^{2}\right) \cos k-\cos \Phi}{i e^{-2 i k} \sin k+\left(1-\gamma^{2}\right) \cos k+\cos \Phi} .
\end{aligned}
$$

From these expressions of the scattering coefficients, we find that the transmission is symmetric or, in other words, $T=$ $T_{\mathrm{L}}=T_{\mathrm{R}}$. Figure 3 illustrates the transmission and reflection probabilities. In the region $k \in(0, \pi)$, the scattering probability is symmetric at $k=\pi / 2$ for a synthetic magnetic flux of $n \pi+$ $\pi / 2(n \in \mathbb{Z})$ [Figs. 3(a)-3(c)], where the scattering spectra are symmetric about the resonance frequency; otherwise (for example, at $\Phi=0$ ), the scattering spectra are asymmetric about
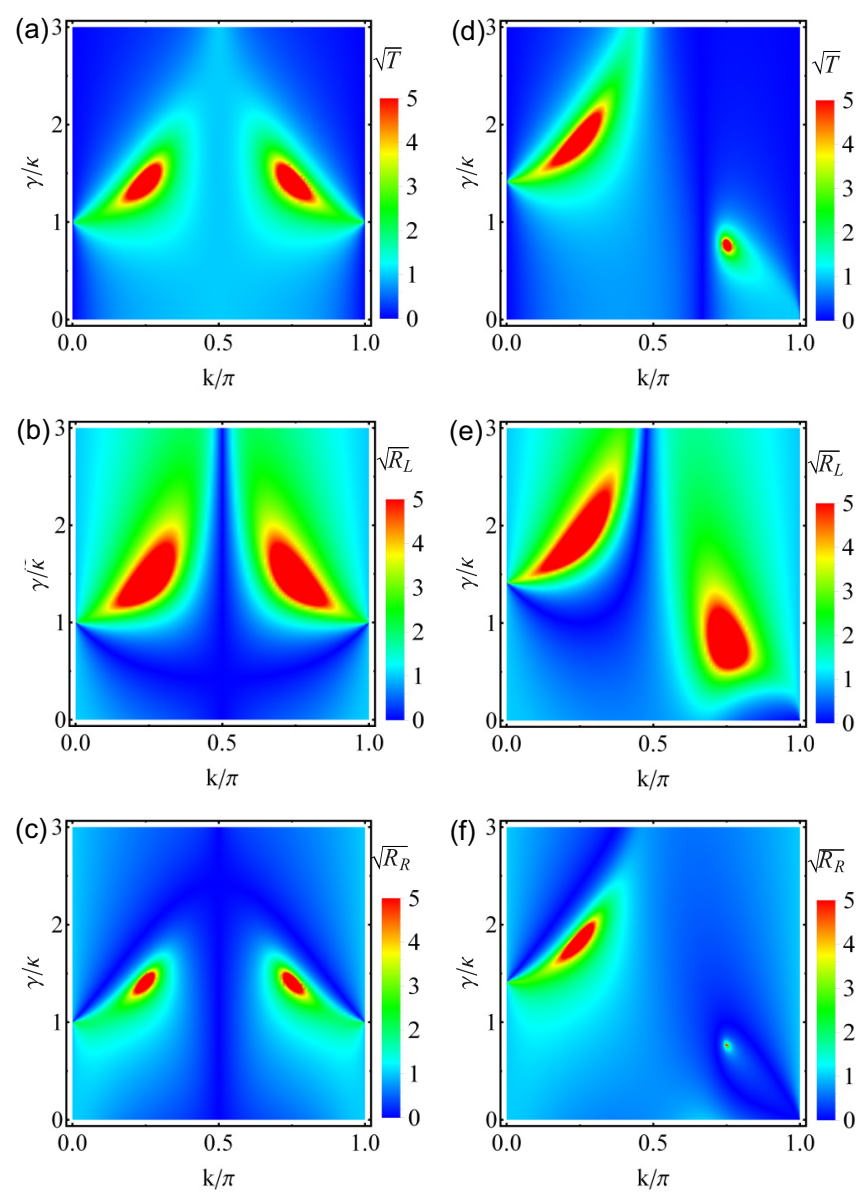

FIG. 3. Transmission and reflection amplitudes. (a)-(c) $\Phi=\pi / 2$ and (d)-(f) $\Phi=0$. (a),(d) Symmetric transmissions $\sqrt{T}$; (b), (e) left reflection $\sqrt{R_{\mathrm{L}}}$; and (c),(f) right reflection $\sqrt{R_{\mathrm{R}}}$.

$k=\pi / 2$ [Figs. 3(d) and 3(f)]. For $\Phi=\pi$, the transmission and reflection probabilities are mirror reflection symmetric about $k=\pi / 2$ at $\Phi=0$. When $k=\pi / 2$, the reflection and transmission are symmetric and $\gamma$ independent, $T_{\mathrm{L}}=T_{\mathrm{R}}=$ $\left(\cos ^{2} \Phi+1\right)^{-1}$ and $R_{\mathrm{L}}=R_{\mathrm{R}}=\left(\cos ^{2} \Phi+1\right)^{-1} \cos ^{2} \Phi$. The synthetic magnetic flux alters the reflection zeros, whereas transmission zeros occur only when $\Phi=n \pi(n \in \mathbb{Z})$. For an input wave vector $k \neq \pi / 2$, the symmetric reflection is broken in the presence of nonzero $\gamma$ even at spectral singularities because the two arms of the interferometer are imbalanced. The reflectionless transmission is unidirectional and synthetic magnetic flux dependent.

Divergence of the scattering coefficients occurs only for wave vectors $k=\pi / 4$ and $3 \pi / 4$ [obtained from Eq. (12)]. Correspondingly, scattering coefficient divergence emerges when the gain and loss rates satisfy $\gamma^{2}=2 \pm \sqrt{2} \cos \Phi$ [obtained from Eq. (13)]. At $k=\pi / 4$, the transmissions ( $t_{\mathrm{L}}$ and $t_{\mathrm{R}}$ ) are larger than zero under any balanced gain and loss $\gamma$ or when the synthetic magnetic flux $\Phi$ is enclosed. Divergence of the scattering coefficients occurs at

$$
\gamma^{2}=\sqrt{2} \cos \Phi+2,
$$

and spectral singularities appear in the region $\sqrt{2-\sqrt{2}} \leqslant$ $|\gamma / \kappa| \leqslant \sqrt{2+\sqrt{2}}$; the spectral singularity curves are shown 

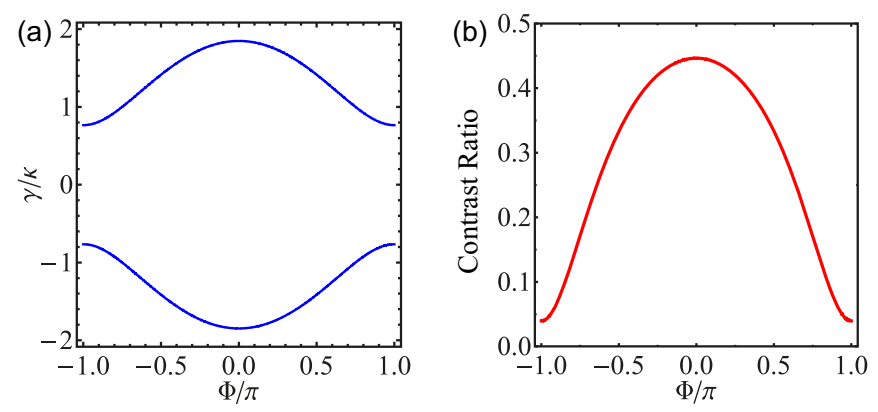

FIG. 4. (a) Spectral singularities in the $\Phi$ and $\gamma$ parameter space for $k=\pi / 4$. The curves indicate the place where scattering coefficients diverge. Spectral singularities appear in the region $\sqrt{2-\sqrt{2}} \leqslant|\gamma / \kappa| \leqslant \sqrt{2+\sqrt{2}}$. (b) The lasing contrast ratio at spectral singularities.

in Fig. 4(a). At spectral singularities, the scattering center acts as a wave emitter [55] and the lasing is asymmetric. The ratio of left-going lasing to right-going lasing, which is obtained by comparing the transmission and reflection coefficients, is plotted in Fig. 4(b). A ratio lower than one indicates that the left side, which includes the gain resonator, has a higher lasing rate. Notably, the three embedded coupled resonators act as a wave emitter at spectral singularities. The lasing is asymmetric, and the contrast ratio of right-traveling wave emission to left-traveling wave emission is

$$
\chi(\Phi)=\frac{\sqrt{2 \sqrt{2} \cos \Phi+4}-1}{\sqrt{2 \sqrt{2} \cos \Phi+4}+1},
$$

which is obtained by comparing the transmission and reflection probabilities. The gain resonator is on the left in this system, and thus left-traveling wave emission is stronger than righttraveling wave emission and the contrast is less than 1 . For example, at $\gamma=\sqrt{2}$ and $\Phi=\pi / 2$, we have $T_{\mathrm{L}} / R_{\mathrm{L}}=1 / 3$ and $T_{\mathrm{R}} / R_{\mathrm{R}}=3$. Notably, $T_{\mathrm{L}} T_{\mathrm{R}}=R_{\mathrm{L}} R_{\mathrm{R}}$ holds independent of the synthetic magnetic flux at spectral singularities, indicating that the asymmetry of the lasing is input-direction independent, whereas the lasing intensity is input-direction dependent. The contrast $T_{\mathrm{L}} / R_{\mathrm{L}}$ decreases as the synthetic magnetic flux $\Phi$ increases from 0 to $\pi$; therefore, the asymmetry of the lasing increases.

A signature of spectral singularities in a wave-packet scattering process is wave emission. We numerically simulate a right-input wave-emission process in the $\mathrm{AB}$ interferometer. The initial excitation is a normalized Gaussian wave packet of $|\Psi(0, j)\rangle=(\sqrt{\pi} / \alpha)^{-1 / 2} \sum_{j} e^{-\left(\alpha^{2} / 2\right)\left(j-N_{\mathrm{c}}\right)^{2}} e^{i k_{\mathrm{c}} j}|j\rangle$ centered at site $N_{\mathrm{c}}$, where $j$ indicates the label of the resonator in the array and $k_{\mathrm{c}}$ is the wave vector of the Gaussian wave packet. At spectral singularities, lasing occurs toward both sides after the wave packet reaches the embedded resonators, and forms an asymmetric plateau described by an error function [55]. The lasing wave intensity increases linearly with time, being proportional to the wave-propagating velocity $2 \kappa \sin k$ and the plateau heights. The time evolution of the Gaussian wave packet is $|\Psi(t, j)\rangle=e^{-i H t}|\Psi(0, j)\rangle$, where $H$ is Hamiltonian of the 1200-site system including both the trimer scattering center $H_{\mathrm{c}}$ and two finite leads connected to the center. In Figs. 5(a), 5(c) and 5(e), a Gaussian wave packet is initially centered at $N_{\mathrm{c}}=900$, the wave vector is $k_{\mathrm{c}}=\pi / 4$, and the packet moves from right to left at a velocity of $\sqrt{2} \kappa$. To emphasize the different asymmetries, the density plots of intensity $P(t, j) / h$ are depicted for comparison, where $P(t, j)=\langle\Psi(t, j) \mid \Psi(t, j)\rangle$ is the intensity of the wave packet and $h$ is the left-traveling wave plateau height in Figs. 5(b), 5(d) and 5(f). At $t \approx 210 / \kappa$, the Gaussian wave packet reaches the scattering center and the intensity begins to increase. Figures 5(b), 5(d) and 5(f) depict the intensity $P(t, j)$ of the lasing at $t=500 / \kappa$ and clearly displays the asymmetric plateau. The left-traveling wave for the right input and the right-traveling wave for the left input induce the same transmitted plateau height $h$, equal to $3 \sqrt{\pi} /(4 \alpha), 3 \sqrt{\pi} /(4 \alpha)$, and $\sqrt{\pi / 10} /(4 \alpha)$ for $\Phi=0, \pi / 2$, and $\pi$, respectively [left plateaus displayed in Figs. 5(b), 5(d) and 5(f)].

Moreover, after the wave packet is scattered by the embedded coupled resonators, the height of the left-traveling wave is $h \chi^{-1}(\Phi)$ and that of the right-traveling wave is $h$ for the left input. By contrast, the height of the left-traveling wave is $h$ and that of the right-traveling wave is $h \chi(\Phi)$ for the right
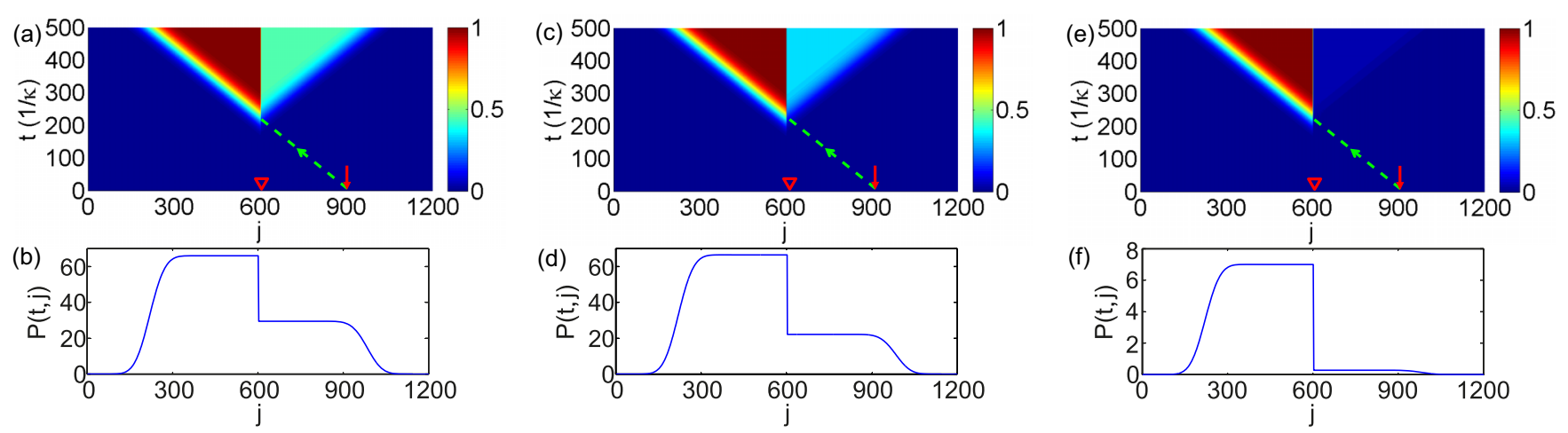

FIG. 5. Numerical simulations of lasing wave intensity for (a),(b) $\Phi=0$, (c),(d) $\Phi=\pi / 2$, and (e),(f) $\Phi=\pi$. (a),(c),(e) Density plots of the intensity $P(t, j) / h$, where $h$ is the left-traveling wave height as shown in (b), (d), and (f) on their left halves. (b),(d),(f) Configuration of the lasing wave intensity $P(t, j)$ at $t=500 / \kappa$. The three coupled resonators embedded in the center of the resonator array are indicated by the red triangles. A Gaussian wave packet with $\alpha=0.02$ and a wave vector $k_{\mathrm{c}}=\pi / 4$ centered at $N_{\mathrm{c}}=900$ (indicated by the red arrow) is initially traveling left. The trajectory of the wave-packet center is indicated by the dashed green line. An asymmetric wave is emitted after reaching the center at approximately $t=210 / \kappa$. 
input. The contour plots in Figs. 5(a), 5(c) and 5(e) indicate that the asymmetry is enhanced as the synthetic magnetic flux is increased from 0 to $\pi$, where the left-traveling wave-emission plateau heights are renormalized to 1 .

\section{CONCLUSION}

A $\mathcal{P} \mathcal{T}$-symmetric two-arm $\mathrm{AB}$ interferometer where three coupled resonators are embedded in a uniformly coupled resonator array is proposed in this paper. Synthetic magnetic flux is enclosed by the three coupled resonators using the pathlength imbalance method. The synthetic magnetic flux acts as a new degree of freedom, controlling light interference but not breaking the $\mathcal{P} \mathcal{T}$ symmetry. In the two-arm $\mathrm{AB}$ interferometer, the $\mathcal{P} \mathcal{T}$ symmetry protects the symmetric transmission; however, the reflection is asymmetric due to the non-Hermitian gain and loss. The conditions for symmetric, asymmetric, and unidirectional lasing at spectral singularities are discussed. The interplay between the synthetic magnetic flux and nonHermiticity controls the lasing at spectral singularities; the lasing is proven asymmetric. The asymmetric lasing at spectral singularities and the reflectionless transmission both vary with the enclosed synthetic magnetic flux. We demonstrate a uniformly coupled $\mathrm{AB}$ interferometer as an illustrative example and show that the asymmetry of lasing is enhanced as the synthetic magnetic flux is increased. Our results could be useful in the design of optical control and lasing devices, and may facilitate the application of $\mathcal{P} \mathcal{T}$-symmetric metamaterials.

\section{ACKNOWLEDGMENTS}

We acknowledge the support of the National Natural Science Foundation of China (Grant No. 11605094) and the Tianjin Natural Science Foundation (Grant No. 16JCYBJC40800).
[1] C. M. Bender and S. Boettcher, Real Spectra in Non-Hermitian Hamiltonians Having $\mathcal{P} \mathcal{T}$ Symmetry, Phys. Rev. Lett. 80, 5243 (1998).

[2] G. Lévai and M. Znojil, Systematic search for $\mathcal{P} \mathcal{T}$-symmetric potentials with real energy spectra, J. Phys. A: Math. Gen. 33, 7165 (2000).

[3] A. Mostafazadeh, Pseudo-Hermiticity versus $\mathcal{P} \mathcal{T}$ symmetry: The necessary condition for the reality of the spectrum of a non-Hermitian Hamiltonian, J. Math. Phys. 43, 205 (2002).

[4] W. D. Heiss, Exceptional points of non-Hermitian operators, J. Phys. A: Math. Gen. 37, 2455 (2004).

[5] A. Ruschhaupt, F. Delgado, and J. G. Muga, Physical realization of $\mathcal{P} \mathcal{T}$-symmetric potential scattering in a planar slab waveguide, J. Phys. A 38, L171 (2005).

[6] K. G. Makris, R. El-Ganainy, D. N. Christodoulides, and Z. H. Musslimani, Beam Dynamics in $\mathcal{P} \mathcal{T}$ Symmetric Optical Lattices, Phys. Rev. Lett. 100, 103904 (2008).

[7] Z. H. Musslimani, K. G. Makris, R. El-Ganainy, and D. N. Christodoulides, Optical Solitons in $\mathcal{P} \mathcal{T}$ Periodic Potentials, Phys. Rev. Lett. 100, 030402 (2008).

[8] S. Klaiman, U. Günther, and N. Moiseyev, Visualization of Branch Points in $\mathcal{P} \mathcal{T}$-Symmetric Waveguides, Phys. Rev. Lett. 101, 080402 (2008).

[9] S. Longhi, Bloch Oscillations in Complex Crystals with $\mathcal{P} \mathcal{T}$ Symmetry, Phys. Rev. Lett. 103, 123601 (2009).

[10] O. Bendix, R. Fleischmann, T. Kottos, and B. Shapiro, Exponentially Fragile $\mathcal{P} \mathcal{T}$ Symmetry in Lattices with Localized Eigenmodes, Phys. Rev. Lett. 103, 030402 (2009).

[11] M. C. Zheng, D. N. Christodoulides, R. Fleischmann, and T. Kottos, $\mathcal{P} \mathcal{T}$ optical lattices and universality in beam dynamics, Phys. Rev. A 82, 010103(R) (2010).

[12] C. Hang, G. Huang, and V. V. Konotop, $\mathcal{P} \mathcal{T}$ Symmetry with a System of Three-Level Atoms, Phys. Rev. Lett. 110, 083604 (2013).

[13] H. Jing, S. K. Ödemir, X.-Y. Lü, J. Zhang, L. Yang, and F. Nori, $\mathcal{P} \mathcal{T}$-Symmetric Phonon Laser, Phys. Rev. Lett. 113, 053604 (2014).

[14] X.-Y. Lü, H. Jing, J.-Y. Ma, and Y. Wu, $\mathcal{P} \mathcal{T}$-Symmetry-Breaking Chaos in Optomechanics, Phys. Rev. Lett. 114, 253601 (2015).
[15] Z.-P. Liu, J. Zhang, S. K. Özdemir, B. Peng, H. Jing, X.-Y. Lü, C.-W. Li, L. Yang, F. Nori, and Y. Liu, Metrology with $\mathcal{P} \mathcal{T}$-Symmetric Cavities: Enhanced Sensitivity Near the $\mathcal{P} \mathcal{T}$-Phase Transition, Phys. Rev. Lett. 117, 110802 (2016).

[16] A. Guo, G. J. Salamo, D. Duchesne, R. Morandotti, M. VolatierRavat, V. Aimez, G. A. Siviloglou, and D. N. Christodoulides, Observation of $\mathcal{P} \mathcal{T}$-Symmetry Breaking in Complex Optical Potentials, Phys. Rev. Lett. 103, 093902 (2009).

[17] C. E. Rüter, K. G. Makris, R. El-Ganainy, D. N. Christodoulides, M. Segev, and D. Kip, Observation of parity-time symmetry in optics, Nat. Phys. 6, 192 (2010).

[18] B. Peng, S. K. Özdemir, F. Lei, F. Monifi, M. Gianfreda, G. L. Long, S. Fan, F. Nori, C. M. Bender, and L. Yang, Parity-timesymmetric whispering-gallery microcavities, Nat. Phys. 10, 394 (2014).

[19] B. Peng, S. K. Özdemir, S. Rotter, H. Yilmaz, M. Liertzer, F. Monifi, C. M. Bender, F. Nori, and L. Yang, Loss-induced suppression and revival of lasing, Science 346, 328 (2014).

[20] L. Chang, X. Jiang, S. Hua, C. Yang, J. Wen, L. Jiang, G. Li, G. Wang, and M. Xiao, Parity-time symmetry and variable optical isolation in active-passive-coupled microresonators, Nat. Photon. 8, 524 (2014).

[21] Z. Zhang, Y. Zhang, J. Sheng, L. Yang, M.-A. Miri, D. N. Christodoulides, B. He, Y. Zhang, and M. Xiao, Observation of Parity-Time Symmetry in Optically Induced Atomic Lattices, Phys. Rev. Lett. 117, 123601 (2016).

[22] L. Feng, Z. J. Wong, R.-M. Ma, Y. Wang, and X. Zhang, Singlemode laser by parity-time symmetry breaking, Science 346, 972 (2014).

[23] H. Hodaei, M.-A. Miri, M. Heinrich, D. N. Christodoulides, and M. Khajavikhan, Parity-time-symmetric microring lasers, Science 346, 975 (2014).

[24] S. Kalish, Z. Lin, and T. Kottos, Light transport in random media with $\mathcal{P} \mathcal{T}$ symmetry, Phys. Rev. A 85, 055802 (2012).

[25] A. Mostafazadeh, Generalized unitarity and reciprocity relations for-symmetric scattering potentials, J. Phys. A 47, 505303 (2014). 
[26] A. Regensburger, C. Bersch, M. A. Miri, G. Onishchukov, D. N. Christodoulides, and U. Peschel, Parity-time synthetic photonic lattices, Nature (London) 488, 167 (2012).

[27] L. Feng, Y.-L. Xu, W. S. Fegadolli, M.-H. Lu, J. E. B. Oliveira, V. R. Almeida, Y.-F. Chen, and A. Scherer, Experimental demonstration of a unidirectional reflectionless parity-time metamaterial at optical frequencies, Nat. Mater. 12, 108 (2013).

[28] Z. Lin, H. Ramezani, T. Eichelkraut, T. Kottos, H. Cao, and D. N. Christodoulides, Unidirectional Invisibility Induced by $\mathcal{P} \mathcal{T}$ Symmetric Periodic Structures, Phys. Rev. Lett. 106, 213901 (2011).

[29] D. L. Sounas, R. Fleury, and A. Alù, Unidirectional Cloaking Based on Metasurfaces with Balanced Loss and Gain, Phys. Rev. Appl. 4, 014005 (2015).

[30] S. Longhi, D. Gatti, and G. D. Valle, Robust light transport in non-Hermitian photonic lattices, Sci. Rep. 5, 13376 (2015).

[31] H. Zhao, S. Longhi, and L. Feng, Robust light state by quantum phase transition in non-Hermitian optical materials, Sci. Rep. 5, 17022 (2015).

[32] A. Mostafazadeh, Spectral Singularities of Complex Scattering Potentials and Infinite Reflection and Transmission Coefficients at Real Energies, Phys. Rev. Lett. 102, 220402 (2009).

[33] S. Longhi, Spectral singularities and Bragg scattering in complex crystals, Phys. Rev. A 81, 022102 (2010).

[34] Z. Ahmed, New features of scattering from a one-dimensional non-Hermitian (complex) potential, J. Phys. A 45, 032004 (2011).

[35] W. D. Heiss, H. Cartarius, G. Wunner, and J. Main, Spectral singularities in $\mathcal{P} \mathcal{T}$-symmetric Bose-Einstein condensates, J. Phys. A 46, 275307 (2013).

[36] X. Liu, S. D. Gupta, and G. S. Agarwal, Regularization of the spectral singularity in $\mathcal{P} \mathcal{T}$-symmetric systems by all-order nonlinearities: Nonreciprocity and optical isolation, Phys. Rev. A 89, 013824 (2014).

[37] Y. D. Chong, L. Ge, H. Cao, and A. D. Stone, Coherent Perfect Absorbers: Time-Reversed Lasers, Phys. Rev. Lett. 105, 053901 (2010).

[38] W. Wan, Y. Chong, L. Ge, H. Noh, A. D. Stone, and H. Cao, Time-reversed lasing and interferometric control of absorption, Science 331, 889 (2011).

[39] Y. Sun, W. Tan, H.-Q. Li, J. Li, and H. Chen, Experimental Demonstration of a Coherent Perfect Absorber with $\mathcal{P} \mathcal{T}$ Phase Transition, Phys. Rev. Lett. 112, 143903 (2014).

[40] S. Longhi, $\mathcal{P} \mathcal{T}$-symmetric laser absorber, Phys. Rev. A 82, 031801(R) (2010).

[41] Y. D. Chong, L. Ge, and A. D. Stone, $\mathcal{P} \mathcal{T}$-Symmetry Breaking and Laser-Absorber Modes in Optical Scattering Systems, Phys. Rev. Lett. 106, 093902 (2011).

[42] C. Hang, G. Huang, and V. V. Konotop, Tunable spectral singularities: coherent perfect absorber and laser in an atomic medium, New J. Phys. 18, 085003 (2016).
[43] K. Fang, Z. Yu, and S. Fan, Photonic Aharonov-Bohm Effect Based on Dynamic Modulation, Phys. Rev. Lett. 108, 153901 (2012).

[44] K. Fang, Z. Yu, and S. Fan, Realizing effective magnetic field for photons by controlling the phase of dynamic modulation, Nat. Photon. 6, 782 (2012).

[45] K. Fang and S. Fan, Effective magnetic field for photons based on the magneto-optical effect, Phys. Rev. A 88, 043847 (2013).

[46] E. Li, B. J. Eggleton, K. Fang, and S. Fan, Photonic AharonovBohm effect in photon-phonon interactions, Nat. Commun. 5, 3225 (2014).

[47] M. Hafezi, E. A. Demler, M. D. Lukin, and J. M. Taylor, Robust optical delay lines with topological protection, Nat. Phys. 7, 907 (2011).

[48] M. C. Rechtsman, J. M. Zeuner, Y. Plotnik, Y. Lumer, D. Podolsky, F. Dreisow, S. Nolte, M. Segev, and A. Szameit, Photonic floquet topological insulators, Nature (London) 496, 196 (2013).

[49] G. Q. Liang and Y. D. Chong, Optical Resonator Analog of a Two-Dimensional Topological Insulator, Phys. Rev. Lett. 110, 203904 (2013).

[50] L. Jin, X. Z. Zhang, G. Zhang, and Z. Song, Reciprocal and unidirectional scattering of parity-time symmetric structures, Sci. Rep. 6, 20976 (2016).

[51] S. Longhi, Non-reciprocal transmission in photonic lattices based on unidirectional coherent perfect absorption, Opt. Lett. 40, 1278 (2015).

[52] X. Q. Li, X. Z. Zhang, G. Zhang, and Z. Song, Asymmetric transmission through a flux-controlled non-Hermitian scattering center, Phys. Rev. A 91, 032101 (2015).

[53] H. Ramezani, Y. Wang, E. Yablonovitch, and X. Zhang, Unidirectional perfect absorber, IEEE J. Sel. Top. Quantum Electron. 22, 115 (2016).

[54] L. Jin, P. Wang, and Z. Song, Unidirectional perfect absorber, Sci. Rep. 6, 32919 (2016).

[55] P. Wang, L. Jin, G. Zhang, and Z. Song, Wave emission and absorption at spectral singularities, Phys. Rev. A 94, 053834 (2016).

[56] H. Ramezani, H. K. Li, Y. Wang, and X. Zhang, Unidirectional Spectral Singularities, Phys. Rev. Lett. 113, 263905 (2014).

[57] M. Hafezi, Synthetic gauge field with photons, Int. J. Mod. Phys. B 28, 1441002 (2014).

[58] M. Hafezi, Measuring Topological Invariants in Photonic Systems, Phys. Rev. Lett. 112, 210405 (2014).

[59] J. D. Joannopoulos, S. G. Johnson, J. N. Winn, and R. D. Meade, Photonic Crystals: Molding the Flow of Light (Princeton University Press, Princeton, 2008).

[60] A. Mostafazadeh and H. Mehri-Dehnavi, Spectral singularities, biorthonormal systems and a two-parameter family of complex point interactions, J. Phys. A 42, 125303 (2009). 